\title{
Influencia del contexto de alto rendimiento deportivo en los vínculos sociafectivos de jugadoras de baloncesto
}

\section{Influence of Contextual Factors in High Performance Sport in Socio-Affective Bonds Among Female Basketball Players}

\section{Influencia do contexto de rendimento alto desportivo nos laços sociafectivos de jugadoras de basquetebol}

\author{
Gloria Ma Díez Flórez, Ma del Carmen Requena Hernández y Marta Zubiaur González
}

Universidad de León

\begin{abstract}
Resumen: El deporte de equipo de alto rendimiento se caracteriza por una futura limitación temporal, la intensidad de contacto físico y social y la temprana edad de inicio. El objetivo de esta investigación es comprobar la influencia de dichas variables en la construcción socioafectiva de jugadoras profesionales en el contexto familiar y deportivo. La muestra se compone de 64 jugadoras profesionales en activo con edades comprendidas entre los 18 y 35 años. El instrumento recoge características sociodemográficas, trayectoria profesional e información de los vínculos socioafectivos dentro y fuera del contexto deportivo y no deportivo. Los análisis estadísticos utilizados han sido descriptivos y el estadístico Chi-cuadrado $\left(\chi^{2}\right)$.Los resultados ponen de manifiesto la inclusión de figuras del contexto familiar y deportivo en su red de apego. Las relaciones de iguales están condicionadas por la edad, la formación y el número de títulos ganados. Las relaciones amorosas de las deportistas aparecen relegadas frente a la familia y la red de iguales. Concluimos que el contexto deportivo de alto rendimiento influye en la construcción socioafectiva de jugadoras de baloncesto profesional.

Palabras clave: baloncesto femenino, rendimiento, apego, amistad, relaciones amorosas.

Abstract: High-performance team sport is characterized for constraints on time horizons, the intensity of physical and social contact, and an early onset age. The aim of this study is to test the influence of these variables in the construction of socio-affective bonds in the familial and sport environments of female professional players. The sample consists of 64 professional players aged between 18 and 35. The instrument collects demographic characteristics, professional background and socio-affective bonding information within and outside the sports environment. The statistical analysis used
\end{abstract}

are both descriptive and Chi-square $\left(\chi^{2}\right)$. The results highlight the inclusion of familial and sport figures in their attachment network. Peer relations are conditioned by age, training and the number of titles won. Romantic relationships are secondary to familial and peer relationships. We conclude that a high-performance sports environment influences the construction of socio-affective bonding among professional women basketball players. Key words: women's basketball, performance, attachment, friendship, romantic relationships.

Resumo: $\mathrm{O}$ esporte de time de rendimento alto é caracterizado por uma limitação futura temporária, a intensidade de exame médico e contato social e o cedo idade de começo. O objetivo desta investigação é conferir a influência destas variáveis no socioafectiva de construção de jugadoras profissional no contexto familiar e desportivo. A amostra está composta de 64 jugadoras profissional dentro ativo com idades entendidas entre os 18 e 35 anos. O instrumento apanha sociodemográficas característico, trajetória profissional e informação do socioafectivos de laços dentro de e fora do esporte e não contexto desportivo. As análises usadas estatísticas foram descritivas e o Chi-quadrado estatístico (.2). Os resultados mostram a inclusão de figuras do contexto familiar e desportivo na rede de anexo deles/delas. As relações de iguala é condicionado pela idade, a formação e o número de títulos ganhados. As relaçôes amorosas dos desportistas parecem banidas em frente à família e a rede de mesmo. Nós concluímos que o contexto desportivo de rendimento alto influencia no socioafectiva de construçáo de jugadoras de basquetebol profissional.

Palavras chave: basquetebol feminino, rendimento, anexo, amizade, relaçóes afectivas.

\section{Introducción}

El desarrollo socioafectivo es una dimensión que hace referencia a la incorporación de cada sujeto que nace a la sociedad donde vive. Tal incorporación supone numerosos procesos

Correspondencia: Gloria Ma Díez Flórez.

Facultad de Ciencias de la Actividad Física y del Deporte. Departamento de Educación Física y Deportiva.

Universidad de león. Campus de Vegazana, s/n. 2407 León. España.

E-mail: gloflo7@hotmail.com; etcgmd00@estudiantes.unileon.es de socialización, la formación de vínculos afectivos, la adquisición de los valores, normas y conocimientos sociales, el aprendizaje de costumbres, roles y conductas que la sociedad transmite y exige cumplir a cada uno de sus miembros y la construcción de una forma personal de ser, porque finalmente cada persona es única (López, 2005, 2009).

El contexto deportivo representa a gran escala los defectos y virtudes de la sociedad, por lo que contamos con un excelente laboratorio donde se pueden analizar comportamien- 
tos normativos de socialización que el deportista establece tanto con las figuras de la disciplina deportiva que practica, entrenador/a y los compañeras del equipo como con las que establece con el contexto no deportivo, familia, red de iguales, pareja (Smith \& Smoll, 1991).

El contexto deportivo de alto rendimiento se compone de unas características idiosincrásicas que van a influir no solo en el rol del deportista sino en su propia construcción personal. En este sentido, en la literatura científica revisada hemos encontrado variables específicas de alto rendimiento que influyen en la formación del deportista, tales como la cohesión de grupo (Jowett, 2009; Lafont, Proeres, \& Vallet., 2007), la motivación (Leo, Sánchez, Sánchez, Amado, \& García, 2009; Moreno, Conte, González, Martín-Albo, \& Núñez, 2010), la autoeficacia (Sáenz-López, Giménez, Ibáñez, \& Jiménez, 2008), el liderazgo (Leite, Vaz, Maçãs, \& Sampaio, 2009; Ruiz, 2006), la ansiedad (Braddock, 2006; Guillén \& Sánchez, 2009) y los estereotipos (Bennett, 2010; Eastman and Billings, 2001; Feu, Ibáñez, Sáenz-López, \& Gutiérrez, 2008; Lin, 2009; Refoyo, Romaris, \& Sampedro, 2009). Investigaciones puntuales se han hecho sobre la contribución de las características del contexto deportivo en el desarrollo personal del deportista. En este sentido destacamos investigaciones realizadas sobre competencia social (Buceta, 1990, 1992, 1998, 2007), la autoeficacia (Sáenz-López, Giménez, Ibáñez, y Jiménez, 2008; Lázaro y Villamarín, 1993) y el rol de género (Krane, 1994, 2001a, 2001b, 2003, 2009; Martín, 2004)

Entre las características específicas del alto rendimiento que pueden ser determinantes en el desarrollo personal de los deportistas de élite, identificamos la temprana edad de comienzo. Éste hecho favorece la discusión del eterno debate no resuelto sobre el peso que tiene la influencia ambiental sobre el desarrollo normativo de las relaciones socioafectivas de la persona. Otra de las características del contexto deportivo de alto rendimiento es la limitación temporal que según Carstensen (1999, 2006,2007,) influye en la priorización de metas tales como el aumento del interés por vivir emociones intensas, selección de las relaciones íntimas y reducción del interés por lo nuevo.

Debido a la escasez de investigaciones relacionadas con el desarrollo personal de la deportista, el objetivo de esta investigación es comprobar la influencia de las variables temporales: temprana edad de comienzo y concavidad temporal, en el desarrollo socioafectivo de jugadoras profesionales.

\section{Método}

\section{Participantes}

La población diana de la muestra fueron las jugadoras de baloncesto espańolas que conformaban la categoría femenina senior. La muestra se compone de 64 jugadoras profesionales de Liga Femenina con edades comprendidas entre los 18 y 35 años, con una edad media de 25,25 (DT=3,71) ańos. Han jugado en todas las categorías anteriores, están en activo y viven fuera del entorno familiar al menos desde hace tres temporadas. Del total de la muestra $6(9,4 \%)$ jugadoras tienen estudios de Educación Secundaria Obligatoria, 13 (20,3\%) jugadoras tienen el título de Bachillerato, 6 (9,4\%) jugadoras han estudiado Formación Profesional y 39 (61\%) jugadoras tiene estudios universitarios. El 100\% de las jugadoras están solteras.

Criterios de inclusión: haber jugado en todas las categorías: alevín, pre-infantil, infantil, cadete, junior, estar en activo en categoría senior o Liga Femenina 2 y Liga Femenina. Criterios de exclusión: vivir en el domicilio familiar, ser amateur y haber jugado menos de dos temporadas en categoría senior.

\section{Instrumento}

El instrumento ha sido elaborado para esta investigación y para ello se ha contado con el asesoramiento y supervisión del experto en el desarrollo de la dimensión sociafectiva Félix López Sánchez, Catedrático de Psicología Afectivo-Sexual, en la Universidad de Salamanca.

El instrumento utilizado se compone de dos partes. La primera se recoge información sobre la trayectoria deportiva de las jugadoras: edad cronológica, edad de inicio en el baloncesto, estudios, motivos de inicio en este deporte, equipos y categorías en los que han jugado, títulos que han ganado, situación deportiva (titulares/no titulares) y sexo de los entrenadores. En la segunda parte se recogen las características de los tres tipos de vínculos: familia, red de iguales y relaciones amorosas, tanto dentro como fuera del ámbito deportivo. En cuanto a las variables relacionadas con el primer tipo de vínculo, la información se organiza en torno al núcleo familiar, donde se pregunta por la relación entre los padres, la relación de los padres con las jugadoras y por lo que esperan de ellas respecto a la forma de vivir en pareja. Además se recoge información sobre el estilo educativo y sus consecuencias. Las variables que componen el estilo educativo son: manifestación de afecto, razonamiento con diálogo, control del afecto y afirmación de poder y las variables recogidas como consecuencias de estos estilos educativos son: alta o baja autoestima, buenas o escasas habilidades sociales, autocontrol, agresividad, recompensa a corto o largo plazo. Por otro lado se recoge información sobre las propiedades que componen los vínculos de apego: deseo de contacto con iguales, confort con el contacto, proximidad, protesta por la separación, interés por lo nuevo y confort con la presencia y el contacto.

En cuanto la red de iguales la información se organiza respecto a las características que definen las relaciones de las amigas de las jugadoras de dentro y fuera del equipo. Las variables analizadas fuera del equipo han sido: la pertenencia 
a una tribu, presión de grupo, diferencias entre el grupo y el equipo deportivo, si han tenido amigas fuera del equipo y su procedencia y sentimientos que priman en sus relaciones. Las variables estudiadas dentro del equipo han sido el desmembramiento del equipo y el sentimiento que genera a las jugadoras cuando se produce, el esfuerzo por mantener el contacto con las compañeras del equipo y éstas con las jugadoras.

Finalmente las variables recogidas en torno al tercer vínculo han sido la jerarquización de las necesidades básicas: familia, red de iguales y relaciones amorosas, y la importancia y apoyo de las figuras de apego.

\section{Procedimiento}

$1^{\text {o }}$ Fase: la primera fase consistió en la elaboración del instrumento sobre la dimensión socioafectiva de las jugadoras de baloncesto de Liga Femenina y Liga Femenina 2. Una vez elaborado se aplicó a cuatro jugadoras profesionales de baloncesto, que no formaron parte de la muestra, para comprobar la adecuación del contenido, corregir errores y considerar las sugerencias de las jugadoras.

$2^{\circ}$ Fase: los datos de esta investigación se recogen durante la temporada 2008/2009. Para la captación de la muestra se contactó con la presidenta de la Asociación de Jugadoras de Baloncesto (AJUB), enviando un e-mail cuyo contenido incluía dos documentos: una carta donde se explicaba las características de la investigación y el instrumento que las jugadoras deberían cumplimentar en formato electrónico, con el fin de que fuera difundido entre las jugadoras asociadas. Este mismo e-mail se envía a los clubes que forman parten de la competición durante esta temporada. $3^{\circ}$ Fase: después de tres meses, fueron remitidos 38 cuestionarios, de los que únicamente 18 fueron válidos, el resto fueron descartados debido a que solamente cumplimentaban los datos de identificación. Tomamos de nuevo contacto con las jugadoras profesionales, esta vez a través de los entrenadores. Para ello nos informamos a través de la Federación de Baloncesto del calendario de partidos de Liga Femenina. Contactamos con los entrenadores de estos equipos vía telefónica y les enviamos por correo electrónico la carta informativa del estudio y el cuestionario, a la vez que se les solicitaba permiso para hablar con las jugadoras. De esta forma obtuvimos 94 cuestionarios, de los que 46 fueron válidos y 48 se invalidaron, por el motivo anteriormente descrito. De los 132 cuestionarios recibidos, 64 han sido válidos para nuestro estudio. La muestra ha sido extraída de una población de 294 jugadoras de baloncesto. Trabajando con un nivel de confianza de 0.89 y un error muestral del $11 \%$.

\section{Análisis estadísticos}

Para el tratamiento de los datos obtenidos del instrumento se han utilizado los estadísticos descriptivos media aritmética, desviación típica, distribución de frecuencias y sus porcentajes. Para la interpretación de la comparación de los datos se ha empleado el estadístico Chi-cuadrado $\left(\chi^{2}\right)$ de Pearson.

\section{Resultados}

Resultados Descriptivos

En la Tabla 1 se recoge información sobre la trayectoria deportiva de las jugadoras de la muestra.

Tabla 1. Datos de trayectoria profesional

\begin{tabular}{lcc}
\hline $\mathbf{N}=\mathbf{6 4}$ & $\mathrm{M}$ & $\mathrm{DT}$ \\
\hline Edad de inicio en el baloncesto & 9,30 & 2,62 \\
Equipos en los que han jugado & 5,30 & 2,34 \\
Títulos ganados & 3,14 & 2,78 \\
\hline $\mathbf{N}=\mathbf{6 4}$ & Frecuencia & Porcentaje \\
\hline MOTIVOS INICIACIÓN BALONCESTO & 3 & 4,7 \\
No contesta & 3 & 10,9 \\
Extra escolar & 7 & 34,4 \\
Familia & 22 & 20,3 \\
Amigos & 13 & 29,7 \\
Gusto por deporte & 19 & \\
CATEGORÍAS DE LOS EQUIPOS & & 45,3 \\
Liga Femenina 2 & 29 & 50,0 \\
Liga Femenina 2 + Liga Femenina & 32 & 4,7 \\
Liga Femenina & 3 & 67,2 \\
TITULAR LA TEMPORADA ANTERIOR & & 32,8
\end{tabular}




\begin{tabular}{lrr}
\hline N $=64$ & M & DT \\
\hline SEXO DEL ENTRENADOR & & \\
Hombre & 54 & 84,4 \\
Mujer & 10 & 15,6 \\
TOTAL & 64 & 100,00 \\
\hline
\end{tabular}

Los resultados de la influencia del contexto deportivo y no deportivo en la construcción del vínculo tipo apego en relación a la convivencia familiar, a los estilos educativos y sus consecuencias se recogen en la Tablas 2 y 3 . Las propiedades y los vínculos de apego, la jerarquización de las necesidades básicas y la importancia y apoyo de los vínculos socioafectivos se muestran en la Tabla 4.

Tabla 2. Características de las figuras de apego

\begin{tabular}{|c|c|c|c|c|c|}
\hline \multicolumn{6}{|l|}{ Características de las figuras de apego } \\
\hline Padres juntos & $\begin{array}{c}\text { SI } \\
90 \%\end{array}$ & $\begin{array}{c}\mathrm{NO} \\
7,8 \%\end{array}$ & $\begin{array}{c}\mathrm{NC} \\
1,6 \%\end{array}$ & & \\
\hline Buena relación pareja padres & $\begin{array}{l}\text { Buena } \\
84,4 \%\end{array}$ & $\begin{array}{l}\text { Mala } \\
14,1 \%\end{array}$ & $\begin{array}{c}\mathrm{NC} \\
1,6 \%\end{array}$ & & \\
\hline Relación cálida de los padres con jugadora & $\begin{array}{l}\text { Buena } \\
92,2 \%\end{array}$ & $\begin{array}{l}\text { Mala } \\
7,8 \%\end{array}$ & $\mathrm{NC}$ & & \\
\hline Mis padres no han sido incondicionales & $\begin{array}{c}\text { De acuerdo } \\
14,1 \%\end{array}$ & $\begin{array}{c}\text { Desacuerdo } \\
82,8 \%\end{array}$ & $\begin{array}{c}\mathrm{NC} \\
3,1 \%\end{array}$ & & \\
\hline Solo uno de mis padres relación cálida & $\begin{array}{c}\text { De acuerdo } \\
87,5 \%\end{array}$ & $\begin{array}{c}\text { Desacuerdo } \\
12,5 \%\end{array}$ & $\begin{array}{l}\text { Madre } \\
87,5 \%\end{array}$ & $\begin{array}{l}\text { Padre } \\
10,9 \%\end{array}$ & $\begin{array}{c}\mathrm{NC} \\
1,6 \%\end{array}$ \\
\hline Mis padres esperan pareja tradicional & $\begin{array}{c}\text { De acuerdo } \\
35,9 \%\end{array}$ & $\begin{array}{c}\text { Desacuerdo } \\
60,9 \%\end{array}$ & $\begin{array}{c}\mathrm{NC} \\
3,1 \% \\
\end{array}$ & & \\
\hline
\end{tabular}

Tabla 3. Estilos educativos y consecuencias contexto familiar y deportivo

\begin{tabular}{|c|c|c|c|c|c|}
\hline \multicolumn{6}{|c|}{ Estilos educativos contexto familiar y deportivo } \\
\hline & Padre & Madre & Entrenador & Todos & NC \\
\hline Manifestación de afecto & $3,1 \%$ & $42,2 \%$ & $0 \%$ & $48,4 \%$ & $6,2 \%$ \\
\hline Razonamiento con diálogo & $12,5 \%$ & $29,7 \%$ & $9,4 \%$ & $37,5 \%$ & $10,9 \%$ \\
\hline Control del afecto & $23,4 \%$ & $17,2 \%$ & $23,4 \%$ & $7,8 \%$ & $28,1 \%$ \\
\hline Afirmación de poder & $26,6 \%$ & $0 \%$ & $37,5 \%$ & $10,9 \%$ & $25 \%$ \\
\hline \multicolumn{6}{|c|}{ Consecuencias estilos educativos contexto familiar y deportivo } \\
\hline & Padre & Madre & Entrenador & Todos & $\mathrm{NC}$ \\
\hline Alta autoestima & $9,4 \%$ & $21,9 \%$ & $7,8 \%$ & $48,4 \%$ & $12,5 \%$ \\
\hline Baja autoestima & $3,1 \%$ & $1,6 \%$ & $18,8 \%$ & $0 \%$ & $76,6 \%$ \\
\hline Buenas habilidades sociales & $7,8 \%$ & $26,6 \%$ & $7,8 \%$ & $48,4 \%$ & $9,4 \%$ \\
\hline Escasas habilidades sociales & $4,7 \%$ & $1,6 \%$ & $7,8 \%$ & $1,6 \%$ & $84,4 \%$ \\
\hline Autocontrol & $15,6 \%$ & $9,4 \%$ & $12,5 \%$ & $37,5 \%$ & $25 \%$ \\
\hline Agresividad/impulsividad & $14,1 \%$ & $6,3 \%$ & $17,2 \%$ & $4,7 \%$ & $57,8 \%$ \\
\hline
\end{tabular}

Tabla 4. Propiedades y vínculos de apego. Necesidades básicas. Importancia figuras de apego

\begin{tabular}{lcccccc}
\hline Propiedades y vínculos de apego & \multicolumn{1}{c}{} & & \\
\hline & Padre & Madre & Entrenador & Equipo & Todos & NC \\
Deseo de contacto con iguales & $1,6 \%$ & $3,1 \%$ & $0 \%$ & $34,4 \%$ & $39,2 \%$ & $21,9 \%$ \\
Confort con el contacto & $3,1 \%$ & $12,5 \%$ & $3,1 \%$ & $9,4 \%$ & $51,8 \%$ & $20,3 \%$ \\
Proximidad & $1,6 \%$ & $12,5 \%$ & $0 \%$ & $12,5 \%$ & $62,5 \%$ & $10,9 \%$ \\
Protesta por la separación & $3,1 \%$ & $9,4 \%$ & $6,3 \%$ & $4,7 \%$ & $17,3 \%$ & $59,4 \%$
\end{tabular}




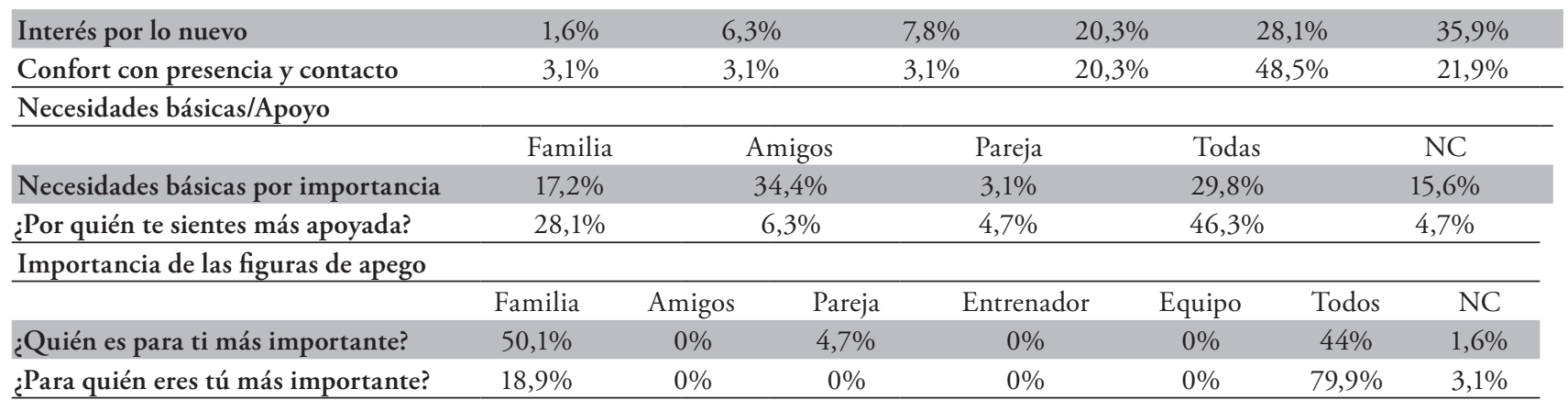

Los resultados de la red de iguales que se muestran en la finen al grupo no deportivo, al grupo deportivo y sus difeTabla 5 , se organizan en relación a las características que de- rencias.

Tabla 5. Datos descriptivos de la red de iguales (Grupo no deportivo y equipo deportivo y diferencias)

\begin{tabular}{|c|c|c|c|c|c|c|c|}
\hline \multicolumn{8}{|l|}{ Grupo no deportivo } \\
\hline Has formado parte de tribus & $\begin{array}{c}\text { SI } \\
14,1 \%\end{array}$ & $\begin{array}{l}\mathrm{NO} \\
82,8 \%\end{array}$ & $\begin{array}{l}\mathrm{NC} \\
3,1 \%\end{array}$ & & & & \\
\hline $\begin{array}{l}\text { Características de la tribu (del 14\% de las } \\
\text { jugadoras) }\end{array}$ & $\begin{array}{c}\text { Compartir } \\
\text { creencias } \\
4,7 \%\end{array}$ & $\begin{array}{c}\text { Apariencia } \\
\text { física } \\
9,4 \%\end{array}$ & $\begin{array}{l}\text { NC } \\
90 \%\end{array}$ & & & & \\
\hline Presión de grupo & $\begin{array}{c}\text { SI } \\
9,4 \%\end{array}$ & $\begin{array}{c}\mathrm{NO} \\
90,6 \%\end{array}$ & & & & & \\
\hline Amigas fuera del equipo deportivo & $\begin{array}{c}\text { SI } \\
93,8 \%\end{array}$ & $\begin{array}{l}\mathrm{NO} \\
4,7 \%\end{array}$ & $\begin{array}{c}\mathrm{NC} \\
1,6 \%\end{array}$ & & & & \\
\hline Procedencia amigas fuera del equipo & $\begin{array}{c}\text { Contexto } \\
\text { escolar } \\
25 \%\end{array}$ & $\begin{array}{c}\text { Actividad } \\
\text { extraescolar } \\
6,3 \%\end{array}$ & $\begin{array}{c}\text { Salidas } \\
\text { nocturnas } \\
4,7 \%\end{array}$ & $\begin{array}{c}\text { Los tres } \\
\text { contextos } \\
57,8 \%\end{array}$ & $\begin{array}{l}\mathrm{NC} \\
9,4 \%\end{array}$ & & \\
\hline Sentimientos positivos en la red de iguales & $\begin{array}{l}\text { Apoyo } \\
10,9 \%\end{array}$ & $\begin{array}{l}\text { Afecto } \\
21,9 \%\end{array}$ & $\begin{array}{l}\text { Confianza } \\
39,1 \%\end{array}$ & $\begin{array}{c}\text { Lealtad } \\
14,1 \%\end{array}$ & $\begin{array}{c}\mathrm{NC} \\
14,1 \%\end{array}$ & & \\
\hline Sentimientos negativos en la red de iguales & $\begin{array}{l}\text { Celos } \\
14,1 \%\end{array}$ & $\begin{array}{c}\text { Enfado } \\
10,9 \%\end{array}$ & $\begin{array}{c}\text { Agresividad } \\
10,9 \%\end{array}$ & $\begin{array}{c}\text { Cólera } \\
6,3 \%\end{array}$ & $\begin{array}{c}\text { Resenti } \\
\text { miento } \\
3,1 \%\end{array}$ & $\begin{array}{l}\text { Tristez } \\
32,8 \%\end{array}$ & $\begin{array}{c}\text { NC } \\
21,9 \%\end{array}$ \\
\hline \multicolumn{8}{|l|}{ Equipo deportivo } \\
\hline \multicolumn{3}{|l|}{ Habitual desmembramiento del equipo } & $\begin{array}{c}\text { SI } \\
20,3 \%\end{array}$ & \multicolumn{2}{|r|}{$\begin{array}{c}\mathrm{NO} \\
79,7 \%\end{array}$} & & \\
\hline \multicolumn{3}{|l|}{ Sentimientos que genera desmembramiento } & $\begin{array}{c}\text { Positivos } \\
4,7 \%\end{array}$ & \multicolumn{2}{|c|}{$\begin{array}{c}\text { Negativos } \\
43,8 \%\end{array}$} & \multicolumn{2}{|r|}{$\begin{array}{c}\mathrm{NC} \\
51,5 \%\end{array}$} \\
\hline \multicolumn{3}{|c|}{ Esfuerzo por mantener contacto con las jugadoras/amigas equipo } & $\begin{array}{c}\text { SI } \\
87,5 \%\end{array}$ & \multicolumn{2}{|r|}{$\begin{array}{c}\mathrm{NO} \\
12,5 \%\end{array}$} & & \\
\hline \multicolumn{3}{|c|}{ Esfuerzo de las jugadoras/amigas por mantener contacto contigo } & $\begin{array}{c}\text { SI } \\
87,5 \%\end{array}$ & \multicolumn{2}{|r|}{$\begin{array}{c}\mathrm{NO} \\
10,9 \%\end{array}$} & \multicolumn{2}{|r|}{$\begin{array}{l}\mathrm{NC} \\
1,6 \%\end{array}$} \\
\hline \multicolumn{8}{|l|}{ Diferencias amigas dentro y fuera del equipo } \\
\hline Res & onsabilidad & Madurez In & $\begin{array}{l}\text { ndependen- } \\
\quad \text { cia }\end{array}$ & $\begin{array}{l}\text { Tiempo } \\
\text { libre }\end{array}$ & $\begin{array}{c}\text { Hábitos } \\
\text { saluda- } \\
\text { bles }\end{array}$ & $\begin{array}{l}\text { Relación } \\
\text { intensa }\end{array}$ & $\begin{array}{l}\text { Relación dura- } \\
\text { dera }\end{array}$ \\
\hline Grupo no deportivo & $24 \%$ & $21,3 \%$ & $19,2 \%$ & $67,7 \%$ & $27,4 \%$ & $23,5 \%$ & $63.9 \%$ \\
\hline Equipo deportivo & $65 \%$ & $63,5 \%$ & $71,4 \%$ & $23 \%$ & $55,6 \%$ & $64,5 \%$ & $21 \%$ \\
\hline NC & $11 \%$ & $15,2 \%$ & $9,4 \%$ & $9,3 \%$ & $17 \%$ & $12,5 \%$ & $15,1 \%$ \\
\hline
\end{tabular}




\section{Datos comparativos}

Los análisis comparativos de la influencia del contexto deportivo y no deportivo en la construcción socioafectiva de los tres vínculos: familia, red de iguales y relaciones amorosas se ilustran en las tablas 6 y 7.

Los datos significativos de los análisis comparativos de los ítems que componen el instrumento de evaluación se muestran en la Tabla 6 y Tabla 7. Cada tabla se divide en dos partes diferenciadas, en la columna de la izquierda aparece la pregunta analizada relacionada con las preguntas con las que ha sido significativa y en la columna de la derecha se registran los datos de significación estadística.

Tabla 6. Influencias en la construcción de la red de apego

\begin{tabular}{|c|c|c|c|c|}
\hline \multirow{2}{*}{\multicolumn{2}{|c|}{ RED DE APEGO }} & \multicolumn{3}{|c|}{$\begin{array}{l}\text { RESULTADOS CHI-CUADRADO } \\
\text { CON SPSS }\end{array}$} \\
\hline & & Valor & $g l$ & $\begin{array}{l}\text { Sig. asintótica } \\
\text { (bilateral) }\end{array}$ \\
\hline \multirow{4}{*}{ 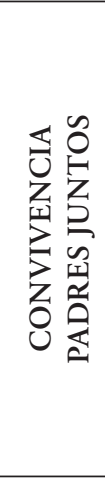 } & $\begin{array}{l}\text { Alta autoestima } \\
\text { Si los padres de las jugadoras están juntos, la consecuencia del estilo educativo de } \\
\text { la madre es la alta autoestima }\end{array}$ & $\begin{array}{c}17,52 \\
4^{\mathrm{a}}\end{array}$ & 8 &, 025 \\
\hline & $\begin{array}{l}\text { Buenas habilidades sociales } \\
\text { Si los padres están juntos, la consecuencia del estilo educativo de la madre es } \\
\text { buenas habilidades sociales }\end{array}$ & $\begin{array}{c}19,38 \\
9^{a}\end{array}$ & 8 & , 013 \\
\hline & $\begin{array}{l}\text { Deseo de contacto con iguales } \\
\text { Si los padres están juntos la característica que mejor define la relación con el equi- } \\
\text { po es el deseo de contacto con iguales. }\end{array}$ & $\begin{array}{c}32,98 \\
7^{a}\end{array}$ & 8 &, 000 \\
\hline & $\begin{array}{l}\text { Interés por lo nuevo } \\
\text { Si los padres están juntos la característica que mejor define la relación con el equi- } \\
\text { po es el interés por lo nuevo. }\end{array}$ & $\begin{array}{c}19,69 \\
7\end{array}$ & 10 &, 032 \\
\hline 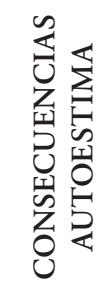 & $\begin{array}{l}\text { Sólo uno de mis padres relación cálida } \\
\text { Cuando la baja autoestima es una consecuencia del estilo educativo del entrenador } \\
\text { tienen una relación cálida con la madre. }\end{array}$ & $\begin{array}{c}18,21 \\
6^{\mathrm{a}}\end{array}$ & 6 & ,006 \\
\hline \multirow{8}{*}{ 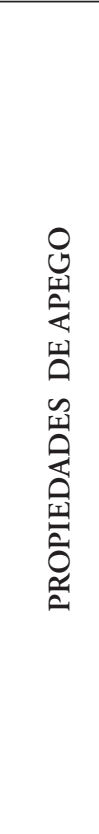 } & $\begin{array}{l}\text { Esfuerzo jugadoras contacto contigo } \\
\text { Cuando la característica que mejor define la relación con el equipo es el deseo de } \\
\text { contacto con iguales, las jugadoras se esfuerzan por mantener el contacto con ella. }\end{array}$ & $\begin{array}{c}40,16 \\
9^{a}\end{array}$ & 8 &, 000 \\
\hline & $\begin{array}{l}\text { Quién es para ti más importante } \\
\text { Cuando la característica que mejor define la relación con el equipo es el deseo de } \\
\text { contacto con iguales, la familia es lo más importante para ellas. }\end{array}$ & $\begin{array}{c}42,55 \\
2^{\mathrm{a}}\end{array}$ & 12 &, 000 \\
\hline & $\begin{array}{l}\text { Cuando la característica que mejor define la relación con el equipo es el interés por } \\
\text { lo nuevo, la familia es lo más importante para ellas. }\end{array}$ & $\begin{array}{c}18,21 \\
6^{\mathrm{a}}\end{array}$ & 6 & ,006 \\
\hline & $\begin{array}{l}\text { Cuando la característica que mejor define la relación con la madre es la protesta } \\
\text { por la separación, la familia es lo más importante para ellas. }\end{array}$ & $\begin{array}{c}26,21 \\
9\end{array}$ & 15 &, 036 \\
\hline & $\begin{array}{l}\text { Para quién eres tú más importante } \\
\text { Cuando la característica que mejor define la relación con el equipo es el deseo de } \\
\text { contacto con iguales, son importantes para la familia. }\end{array}$ & $\begin{array}{c}19,28 \\
3^{\mathrm{a}}\end{array}$ & 8 & ,013 \\
\hline & $\begin{array}{l}\text { Sentimiento desmembramiento equipo } \\
\text { Cuando la característica que mejor define la relación con la madre es el confort } \\
\text { con el contacto, el sentimiento que produce el desmembramiento es tristeza. }\end{array}$ & $\begin{array}{c}57,83 \\
5^{a}\end{array}$ & 30 & ,002 \\
\hline & $\begin{array}{l}\text { Cuando la característica que mejor define la relación con el entrenador es el interés } \\
\text { por lo nuevo, el sentimiento que genera el desmembramiento es tristeza. }\end{array}$ & $\begin{array}{c}46,50 \\
3^{\mathrm{a}}\end{array}$ & 30 &, 028 \\
\hline & $\begin{array}{l}\text { Desmembramiento equipo habitual } \\
\text { Cuando la característica que mejor define la relación con el equipo es el interés por } \\
\text { lo nuevo, no es habitual el desmembramiento del equipo. }\end{array}$ & $\begin{array}{c}11,94 \\
4^{\mathrm{a}}\end{array}$ & 5 &, 036 \\
\hline
\end{tabular}


Tabla 7. Influencias en la construcción de la red de iguales

\begin{tabular}{|c|c|c|c|c|}
\hline \multirow{2}{*}{\multicolumn{2}{|c|}{ RED DE IGUALES }} & \multicolumn{3}{|c|}{$\begin{array}{l}\text { RESULTADOS CHI-CUADRADO } \\
\text { CON SPSS }\end{array}$} \\
\hline & & \multirow{2}{*}{$\begin{array}{c}\text { Valor } \\
6,94 \\
4^{\mathrm{a}}\end{array}$} & \multirow{2}{*}{$\begin{array}{l}g l \\
2\end{array}$} & \multirow[t]{2}{*}{$\begin{array}{l}\text { Sig. asintótica } \\
\text { (bilateral) }\end{array}$} \\
\hline 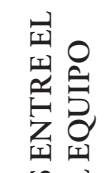 & $\begin{array}{l}\text { Edad } \\
\text { Han encontrado diferencias entre el grupo y el equipo las jugadoras de menor } \\
\text { edad, frente a las de mayor edad que no las han encontrado. }\end{array}$ & & & \\
\hline 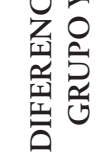 & $\begin{array}{l}\text { Estudios } \\
\text { Han encontrado diferencias las jugadoras universitarias, frente a las no universita- } \\
\text { rias que no las han encontrado. }\end{array}$ & $\begin{array}{c}22,28 \\
8^{\mathrm{a}}\end{array}$ & 8 &, 004 \\
\hline \multirow{11}{*}{ 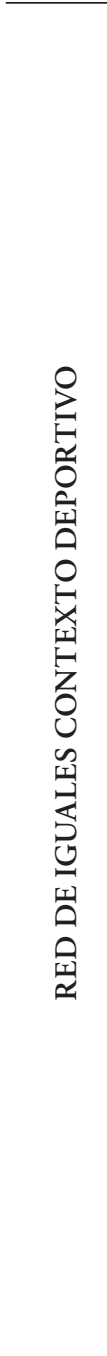 } & $\begin{array}{l}\text { Títulos ganados (BI,6) } \\
\text { Cuando han tenido amigas fuera del equipo han ganado más títulos. }\end{array}$ & $\begin{array}{c}9,55 \\
9^{a}\end{array}$ & 4 & ,049 \\
\hline & $\begin{array}{l}\text { Características tribus } \\
\text { Para las jugadoras que se han esforzado por mantener el contacto con las } \\
\text { jugadoras-amigas del equipo, la característica de las tribus a las que pertenecían es } \\
\text { compartir creencias. }\end{array}$ & $\begin{array}{c}7,29 \\
8^{\mathrm{a}}\end{array}$ & 2 & ,026 \\
\hline & $\begin{array}{l}\text { Cuando se esfuerzan las jugadoras-amigas por mantener el contacto con ellas, las } \\
\text { jugadoras no han pertenecido a tribus. } \\
\text { Cuando no se esfuerzan las jugadoras-amigas por mantener el contacto con ellas, } \\
\text { han pertenecido a alguna tribu. }\end{array}$ & $\begin{array}{c}36,81 \\
9^{\mathrm{a}}\end{array}$ & 4 &, 000 \\
\hline & $\begin{array}{l}\text { Cuando han tenido amigas fuera del equipo, no han formado parte de ninguna } \\
\text { tribu. }\end{array}$ & $\begin{array}{c}32,44 \\
6^{a}\end{array}$ & 4 &, 000 \\
\hline & $\begin{array}{l}\text { Cuando han tenido amigas fuera del equipo la característica de la tribu a la que } \\
\text { pertenecían era la apariencia física. } \\
\text { Cuando no han tenido amigas fuera del equipo la característica de la tribu a la que } \\
\text { pertenecían era compartir creencias }\end{array}$ & $\begin{array}{c}20,92 \\
2^{\mathrm{a}}\end{array}$ & 4 &, 000 \\
\hline & $\begin{array}{l}\text { Sentimientos positivos relaciones iguales } \\
\text { Cuando el sentimiento que producía el desmembramiento es tristeza, el sentimien- } \\
\text { to positivo que prima en sus relaciones con iguales es confianza. }\end{array}$ & $\begin{array}{c}42,55 \\
8^{\mathrm{a}}\end{array}$ & 24 &, 011 \\
\hline & $\begin{array}{l}\text { Necesidades básicas } \\
\text { Las jugadoras que se han esforzado por mantener el contacto con las jugadoras- } \\
\text { amigas del equipo, valoran en primer lugar por orden de importancia la red de } \\
\text { iguales. }\end{array}$ & $\begin{array}{c}17,02 \\
1^{\mathrm{a}}\end{array}$ & 4 &, 002 \\
\hline & $\begin{array}{l}\text { Cuando se esfuerzan las jugadoras-amigas por mantener el contacto con ellas, } \\
\text { valoran en primer lugar por orden de importancia la red de iguales. }\end{array}$ & $\begin{array}{l}20,47 \\
99^{a}\end{array}$ & 8 & ,009 \\
\hline & $\begin{array}{l}\text { Por quién te sientes más apoyada } \\
\text { Cuando el sentimiento que producía el desmembramiento es tristeza, se sienten } \\
\text { más apoyadas por la familia }\end{array}$ & $\begin{array}{c}44,70 \\
6^{\mathrm{a}}\end{array}$ & 24 & ,006 \\
\hline & $\begin{array}{l}\text { Cuando han tenido amigas fuera del equipo se sienten más apoyadas por la fami- } \\
\text { lia. }\end{array}$ & $\begin{array}{c}17,60 \\
0^{\mathrm{a}}\end{array}$ & 8 &, 024 \\
\hline & $\begin{array}{l}\text { Cuando se esfuerzan las jugadoras-amigas por mantener el contacto con ellas, se } \\
\text { sienten más apoyadas por la familia. }\end{array}$ & $\begin{array}{c}17,42 \\
9^{a}\end{array}$ & 8 &, 026 \\
\hline
\end{tabular}




\section{Discusión}

Los resultados de esta investigación muestran que las jugadoras incluyen al equipo en las propiedades de apego y a los padres y al entrenador como agentes en las consecuencias de los estilos educativos. El desmembramiento y la temporalidad de las amigas dentro del equipo caracterizan la red de iguales. Las relaciones amorosas tienen una importancia relativa en las redes socioafectivas.

En cuanto al apego, primera red socioafectiva, los padres forman una pareja convencional, hombre y mujer, manteniendo una relación cálida e incondicional entre ellos y respecto a las jugadoras. Las características de apego: el deseo de contacto con iguales, confort con la presencia y el contacto, proximidad e interés por lo nuevo se identifican con el equipo. Datos que se justifican por la oportunidad de convivencia entre las compañeras del equipo, dado que se inician de forma temprana en el baloncesto, empiezan alrededor de los nueve años, y por el tiempo e intensidad que dedican al entrenamiento.

En cuanto a las características de los estilos educativos, manifestación de afecto y razonamiento con diálogo y las consecuencias como la alta autoestima y las buenas habilidades sociales, se asocian con la madre. Paralelamente, los estilos relacionados con el control del afecto y la afirmación de poder cuyas consecuencias se relacionan con el autocontrol, la agresividad e impulsividad se asocian con las figuras masculinas del padre y del entrenador. La asignación de roles sigue un patrón convencional por lo tanto no justificaría que la convivencia del contexto deportivo y no deportivo influya en el modelo educativo de las jugadoras. En todo caso ratificaría comportamientos estancos en función del género.

En cuanto a la red de iguales las jugadoras de menor edad y con titulación universitaria manifiestan que hay diferencias entre las amigas dentro y fuera del equipo. Las deportistas constatan que el equipo se caracteriza por relaciones con mayor madurez, responsabilidad e independencia que los grupos no deportivos. Hacen hincapié en que su vida de adolescente no sigue la norma en cuanto a salidas nocturnas, tiempo de ocio (limitado), hábitos de vida saludables (en el grupo aparecen el tabaco y las drogas) y consideran que las relaciones son menos duraderas y más intensas con las amigas de dentro del equipo que de fuera del equipo.
Por otro lado todas ellas afirman que han tenido amigas tanto dentro como fuera del equipo en la etapa de la niñez y de la adolescencia. La procedencia de las amigas en la etapa de la niñez ha sido el contexto escolar y las actividades extraescolares, mientras que en la etapa de la adolescencia ha sido fundamentalmente las salidas nocturnas. El sentimientos positivo que destacan las jugadoras en la relación con las amigas de fuera del equipo es la confianza y como negativo la tristeza. Sentimiento que prevalece también cuando se da el desmembramiento de las compañeras dentro del equipo. Sin embargo únicamente hacen esfuerzo por mantener el contacto con la amigas de fuera del equipo cuando no han pertenecido a una tribu o han ganado más títulos. Finalmente, tanto si el esfuerzo de contacto ha sido por parte de la jugadora como por parte de las compañeras, han antepuesto al equipo y a la familia por delante de la pareja en la prioridad de las necesidades básicas y en el apoyo percibido. Este dato es llamativo dado que todas las jugadoras tienen pareja. En este sentido los datos normativos de la literatura revisada recogen que los sujetos, en edades similares a los de nuestra investigación, priorizan en las necesidades básicas a la pareja por delante de la familia y los iguales (López, 1999).

Las características de temporalidad e intensidad de contacto de la práctica deportiva del alto rendimiento justificaría la prioridad que han establecido en sus necesidades básicas. En este sentido Carstensen (1999, 2006, 2007) pone de manifiesto en su teoría socioemocional selectiva que cuando las personas ven limitado el tiempo de contacto tienden a realizar una poda emocional en las relaciones socioafectivas. Esto es, priorizan las relaciones cercanas, íntimas y duraderas porque les proporciona bienestar y seguridad. La conclusión de nuestra investigación nos lleva a poner de manifiesto que la temprana edad de comienzo en el contexto deportivo influye en lo vínculos socioafectivos de las jugadoras de baloncesto de élite, dado que incluyen al entrenador y al equipo en el vínculo de apego. Por otro lado la concavidad temporal del alto rendimiento justificaría la prioridad de la familia en las relaciones socioafectivas de las jugadoras.

Se propone que en futuras investigaciones se constate la veracidad de estos resultados con mayor tamańo muestral y otras disciplinas deportivas de alto rendimiento.

\section{Referencias}

Bennett, S. (2010). Why She Plays: The World of Women's Basketball (Book Review). Women in Sport \& Physical Activity 22, $72-73$.

Braddock, A. E. (2006). Expectations and self-focused attention in sport performance anxiety, Braddock, Autumn Elisabeth: U California, Los Angeles, US.

Buceta, J.M. (1990). Aspectos psicológicos a tener en cuenta en relación con las deportistas españolas de alta competición. Actas del Seminario "Mujer y Deporte" (17 al 19 de mayo de 1990). Ministerio de Educación y Ciencia.
Buceta, J.M. (1992) Intervención psicológica con el equipo nacional olímpico de baloncesto femenino. Revista de Psicología del Deporte, 2, 69-87. Buceta, J.M. (1998). Psicología del entrenamiento deportivo. Madrid: Dykinson.

Buceta, J. M. (2007). Psicología del deporte: nuevas aplicaciones. Conferencia en la X Jornadas de actualización en Psicología del Deporte. [Vídeo en línea]. Disponible: http//palestraweb.com. Formación en Psicología del Deporte y Entrenamiento Deportivo de la UNED. Madrid: UNED.

Carstensen, L. L., Isaacowitz, D. M., \& Charles, S. T. (1999). Taking time 
seriously: A theory of socioemotional selectivity. American Psychologist, 54, 165-181.

Carstensen, L.L. (2006). The influence of a sense of time on human development. Science, 312, 1913-1915.

Carstensen, L. L. (2007). Growing old or living long: Take your pick. Issues in Science and Technology, Winter 2007, 41-50.

Eastman, S.T., \& Billings, A.C. (2001). Biased voices of sports: Racial and gender stereoptyping in college basketball announcing. Howard Journal of Communications 12(4), 183-201.

Feu, S., Ibáñez, S.J., Sáenz-López, \& Gutiérrez, (2008). Evolución de las jugadoras en las selecciones españolas de baloncesto. Apunts. Educación Física y Deportes 93, 71-78.

Guillén, F., \& Sánchez, R. (2009). Competitive anxiety in expert female athletes: Sources and intensity of anxiety in National Team and First Division Spanish basketball players. Perceptual and Motor Skills 109(2), 407-419.

Jowett, S. (2009). Validating Coach-Athlete Relationship Measures with the Nomological Network. Measurement in Physical Education and Exercise Science, 13, 34-51.

Krane, V. (1994). A Feminist Perspective on Contemporary Sport. The Sport Psychologist, 8, 393-410.

Krane, V. (2001a). "We can be athletic and feminine," but do we really want to? Challenging hegemonic femininity in women's sport. Quest, 53, 115-133.

Krane, V. (2001b). One lesbian feminist epistemology: Integrating feminist standpoint, queer theory, and feminist cultural studies. The Sport Psychologist, 15, 401-411.

Krane, V., \& Barber, H. (2003). Lesbian experiences in sport: A social identity perspective. Quest, 55, 328-346.

Krane, V. (2009). A sport odyssey. Qualitative Research in Sport and Exercise, 1(3), 221-238.

Lafont, L., Proeres, M., \& Vallet, C. (2007). Cooperative group learning in a team game: Role of verbal exchanges among peers. Social Psychology of Education, 10(1), 93-113.

Leite, N., Vaz, L., Maçãs, V., \& Sampaio, J. (2009). Coaches perceived importance of drills items in basketball players' long-term development.
Revista de Psicología del Deporte, 18, (Supl.), 457-461.

Lázaro, I. y Villamarín, F. (1993). Capacidad predictiva de la auto-eficacia individual y colectiva sobre el rendimiento en jugadoras de baloncesto. Revista De Psicología Del Deporte, 4, 27-38.

Leo, F.M., Sánchez, P.A., Sánchez, D., Amado, D., \& García Calvo, T. (2009). Influence of the motivational climate created by coach in the sport commitment in youth basketball players. Revista de Psicologia del Deporte 18(Supl.), 375-378.

Lin, C.S. (2009). Gender differences in spectator's motivation for Taiwanese men's basketball. Lin, Cheng-Shiun: United States Sports Academy, US

López, F. (1999). Evolución del apego desde la adolescencia hasta la muerte. En López F., Etxebarría I., Fuentes M.J. y Ortiz M.J. (Eds.), Desarrollo afectivo y social (pp.41-66). Psicología. Pirámide.

López Sánchez, F., Etxebarria Bilbao, I., Fuentes Rebollo, M.J., Ortiz, M.J (coord.) (2005). Desarrollo afectivo y social. Madrid: Pirámide.

López, F. (2009). Amores y desamores. Procesos de vinculación y desvinculación sexuales y afectivos. Madrid: Biblioteca Nueva.

Martín, M. (2004). Generating female freedom among women's relationships in rugby union narratives of sexual difference. Tesis doctoral no publicada, Brunel University, London.

Moreno, J.A., Conte L., González, D., Martín-Albo, J., \& Núñez, J.L. (2010). Efectos de intervención del clima tarea sobre la motivación de estudiantes en la enseńanza deportiva. Estudios de Psicología, 31(1), 6777.

Refoyo, I., Romaris, I.U., \& Sampedro, J. (2009). Analysis of men's and women's basketball fast-breaks. Revista de Psicología del deporte, 18(3), 439-444.

Sáenz-López, P., Giménez, F.J., Ibáñez, S.J., \& Jiménez, A.C. (2008). La visión de las jugadoras internacionales de baloncesto sobre su proceso de formación. Habilidad Motriz, 31, 33-42.

Ruiz, R. (2006). Diferencias de liderazgo en entrenadores de judo a nivel competitivo. Cuadernos de Psicología del Deporte, 6(2), 21-38.

Smith, R.E., \& Smoll, F.L. (1991). Behavioral research and intervention in youth sports. Behaviour Therapy, 22, 329-344. 